\title{
Strong Absorption model for the studying of Pion-Nuclei Elastic Scattering
}

\author{
Rony Mallick ${ }^{1}$, Dr. Dipika Rani Sarker ${ }^{2}$ \\ ${ }^{l}$ (Department. of Physics, Jessore University of Science and Technology, Jessore-7408, Bangladesh) \\ ${ }^{2}$ (Department of Physics, Jagannath University, Dhaka, Bangladesh)
}

\begin{abstract}
The angular distribution of pions, elastically scattered from a number of nuclei between ${ }^{6} \mathrm{Li}$ and ${ }^{118} \mathrm{Sn}$ are studied in terms of the strong absorption model of Frahn and Venter[1-2] using the three parameters version of this model. We find that a reasonably good description to the angular distribution of experimental elastic scattering data is possible. The best fit parameters of the model are the cut-off angular momentum $\Lambda$, the rounding parameter $\Delta$ and the real nuclear phase shift $\mu$. The interaction radius $R$, the surface diffuseness $d$ and the reaction cross-section $\sigma_{R}$ are determined from these parameter values. From such an analysis, the SAM parameters are unique whereas the optical model parameters suffer from discrete and continuous ambiguities and that is why the parameters of the optical model are not unique.
\end{abstract}

Keywords: Nuclear reactions and scattering, optical and diffraction model, elastic pion scattering.

\section{Introduction}

The strong absorption model (SAM), as alternative to the optical model, where the potential concept is replaced by the direct parameterization of the scattering function $\eta_{l}$, was introduced by Frahn and Venter for the studying of the interaction of the strongly absorbed nuclear probes (hadrons and various other nuclear particles) with nuclei. One of the most important means of obtaining information about the nuclear properties is the scattering of nuclear particles. The scattering of pions $\left(\pi^{ \pm}\right)$have been playing a very vital role in nuclear physics. It is difficult to correlate the experimental data directly with the properties of the fundamental nuclear interactions, since the scattering of complex nuclei represent a complicated many body problem. This is why simplifying assumption (nuclear models) have been suggested which connect certain average features of many body problems that are connected directly with measurable quantities. During the past several years numerous analyses of the elastic and inelastic scattering data of different projectiles have been carried out using the SAM (strong absorption model) formalism. These measurements have provided a large amount of information of interest regarding the size, diffuseness, deformation and other properties of nuclei. A larger member of experiments and analyses of the elastic scattering of different incident particles at different projectile energies from various target nuclei have been performed through the SAM formalism in order to study the parameters variation over a wide range of conditions. The Strong Absorption Model of Frahn and Venter [2] is successful in analyzing scattering data. SAM analyses of different nucleus elastic scattering data are of important, since they provide us with parameters required in analyzing various non-elastic angular distributions. The present work has been under taken on the elastic scattering of pions $\left(\pi^{ \pm}\right)$at different projectile energy $163-500 \mathrm{MeV}$. Various angular distribution data on the elastic scattering are from Refs. [3-4].

\section{The SAM Formalism}

The strong absorption model starts with a direct parametrization of the scattering function $\eta_{1}$ as given by,

$$
\eta_{l} \exp \left(-2 \mathrm{i} \sigma_{l}\right)=\mathrm{g}(\lambda)+\mathrm{i} \mu \frac{d g(\lambda)}{\lambda}
$$

Where $\sigma_{l}$ is the Coulomb phase shift for the $l$ - the partial wave and $\mathrm{g}(\lambda)$ is a continuous monotonic function of the angular momentum. A convenient form of $g(\lambda)$ is the Woods - Saxon form, namely,

$$
\mathrm{g}(\lambda)=[1+\exp \{(\Lambda-\lambda) / \Delta\}]^{-1}
$$

Here, $\Lambda$ is the cut-off angular momentum which just grazes the nuclear surface and $\Delta$ is the rounding parameter. These are related semi-classically to the interaction radius $\mathrm{R}$ and the surface diffuseness $\mathrm{d}$, respectively through the expressions

$$
\Lambda=\mathrm{k} R\left(1-\frac{2 n}{k R}\right)^{1 / 2}
$$

And

$$
\Delta=\mathrm{kd}\left(1-\frac{n}{k R}\right)\left(1-\frac{2 n}{k R}\right)^{-1 / 2}
$$

Where, $\mathrm{n}$ and $\mathrm{k}$ are respectively the Coulomb parameter and the wave number.

Austern and Blair[5] has shown the generalized scattering amplitude of adiabatic distorted wave theory can be expressed in terms of the derivatives of elastic amplitude. The strong absorption model form of $\eta_{1}$ was 
introduced by Potgieter and Frahn [6] in the generalized scattering amplitude derived by Austern and Blair [5] and a closed form of expression for inelastic scattering cross-section is obtained in terms of the three parameters namely $\Lambda, \Delta$ and $\mu$ all are fixed from the elastic scattering analysis. The fourth parameter proportional to the reduced matrix element of inelastic interaction is obtained from a normalization $\mathrm{N}$ of the theory to experiment. One can obtain the deformation length $\delta_{\mathrm{L}}$ of the target nucleus from collective excitation from such normalization and hence the deformation parameter $\beta_{\mathrm{L}}$ of the different multipole modes can be determined.

\section{Results And Discussions}

The We have analyzed the elastic scattering of pions $\left(\pi^{ \pm}\right)$from various nuclei ${ }^{6} \mathrm{Li},{ }^{12} \mathrm{C},{ }^{40,44} \mathrm{Ca},{ }^{90} \mathrm{Zr}$ and ${ }^{118} \mathrm{Sn}$ over the energy range of $163-500 \mathrm{MeV}$ on the basis of strong absorption model (SAM) due to Frahn and Venter [1-2].The analyses of experimental data are carried out by a systematic variation of the SAM parameters based on the criterion of minimum root square difference between the experimental and theoretical crosssection. The obtained results of the SAM analysis rendering the best fit parameter values are presented in tables (1 and 2). The experimental data along with the theoretically calculated angular distributions are graphically shown in Figs.1-8.The angular distributions are quite satisfactory and are of similar nature achieved with that of the optical model and other models [7-8] characterized by these latter methods have at least six parameters as against only three parameters in the SAM.

The SAM parameters $\Lambda, \Delta$ and $\mu$ are uniquely given by the analysis. The cut-off angular momentum $\Lambda$ increases smoothly with the increase in target mass number while the beam energy remains same, shown in tables 1 and 2. The rounding parameter $\Delta$ increases with the increase in projectile energy and target mass number. The parameter $\Delta$, controls the average slope of the angular distributions.

Table. 1. SAM Parameters for the positive pion $\left(\pi^{+}\right)$.

\begin{tabular}{|c|c|c|c|c|c|c|c|c|c|}
\hline \multirow{2}{*}{$\begin{array}{l}\text { Serial } \\
\text { No. }\end{array}$} & \multirow{2}{*}{$\begin{array}{l}\text { Target } \\
\text { Nucleus }\end{array}$} & \multirow{2}{*}{$\begin{array}{l}\text { Beam } \\
\text { Energy } \\
(\mathrm{MeV})\end{array}$} & \multicolumn{4}{|c|}{ SAM Parameter } & \multicolumn{3}{|c|}{ Derived quantities } \\
\hline & & & $\Lambda$ & $\Delta$ & $\mu$ & $\mu / 4 \Delta$ & $\mathrm{R}(\mathrm{fm})$ & $\mathrm{d}(\mathrm{fm})$ & $\sigma_{\mathrm{R}(\mathrm{mb})}$ \\
\hline 1 & ${ }^{6} \mathrm{Li}$ & 240 & $4 \times 10$ & $0 * 94$ & $0 * 60$ & $0^{*} 16$ & $2 * 53$ & $0 * 58$ & $31^{*} 884$ \\
\hline 2 & ${ }^{40} \mathrm{Ca}$ & 292.5 & $9 * 37$ & $1 * 10$ & $1^{*} 48$ & $0^{*} 34$ & $4^{*} 67$ & $0 * 54$ & $80^{*} 229$ \\
\hline 3 & ${ }^{44} \mathrm{Ca}$ & 180 & $7 * 20$ & $0 * 80$ & $0 * 40$ & $0^{*} 13$ & $5 * 11$ & $0 * 55$ & $98=201$ \\
\hline 4 & ${ }^{90} \mathrm{Zr}$ & 163 & $8^{*} 00$ & $0 * 70$ & $0 * 60$ & $0^{*} 02$ & $6 * 15$ & $0 * 52$ & $129^{*} 13$ \\
\hline 5 & ${ }^{90} \mathrm{Zr}$ & 400 & $13^{*} 70$ & $1 * 25$ & $1^{*} 95$ & $0 * 39$ & $5 * 35$ & $0 * 48$ & $97^{*} 67$ \\
\hline 6 & ${ }^{90} \mathrm{Zr}$ & 500 & $16^{*} 10$ & $1^{*} 60$ & $0^{*} 90$ & $0 * 14$ & $5 * 24$ & $0 \times 51$ & $101^{*} 47$ \\
\hline 7 & ${ }^{118} \mathrm{Sn}$ & 163 & $8 * 50$ & $0 * 70$ & $0=28$ & $0^{*} 10$ & 657 & $0 * 52$ & $144^{*} 66$ \\
\hline
\end{tabular}

Table. 2. SAM Parameters for the negative pion $\left(\pi^{-}\right)$.

\begin{tabular}{|c|c|c|c|c|c|c|c|c|c|}
\hline \multirow{2}{*}{$\begin{array}{l}\text { Serial } \\
\text { No. }\end{array}$} & \multirow{2}{*}{$\begin{array}{l}\text { Target } \\
\text { Nucleus }\end{array}$} & \multirow{2}{*}{$\begin{array}{l}\text { Beam } \\
\text { Energy } \\
(\mathrm{MeV})\end{array}$} & \multicolumn{4}{|c|}{ SAM Parameter } & \multicolumn{3}{|c|}{ Derived quantities } \\
\hline & & & $\Lambda$ & $\Delta$ & $\mu$ & $\mu / 4 \Delta$ & $\mathrm{R}(\mathrm{fm})$ & $\mathrm{d}(\mathrm{fm})$ & $\sigma_{\mathrm{R}}(\mathrm{mb})$ \\
\hline 1 & ${ }^{6} \mathrm{Li}$ & 240 & $4 * 3$ & $1^{*} 00$ & $0^{*} 60$ & $0 * 15$ & $2 \times 63$ & $0^{*} 61$ & $35^{*} 40$ \\
\hline 2 & ${ }^{12} \mathrm{C}$ & 200 & $4: 8$ & $0 * 98$ & $0^{*} 40$ & $0 * 10$ & $3 * 16$ & $0^{*} 65$ & $49^{*} 18$ \\
\hline 3 & ${ }^{12} \mathrm{C}$ & 260 & $4 * 9$ & $0 * 90$ & $0^{*} 58$ & $0 * 28$ & $2 * 69$ & $0^{*} 49$ & $33^{*} 69$ \\
\hline 4 & ${ }^{40} \mathrm{Ca}$ & $292 * 5$ & $9=8$ & $1 * 40$ & $0^{*} 95$ & $0 * 17$ & $4 * 73$ & $0 * 69$ & 96.59 \\
\hline 5 & 每4 $\mathrm{Ca}$ & 180 & $7 * 2$ & $0 * 80$ & $0^{*} 04$ & $0^{*} 01$ & $4: 88$ & $0^{*} 55$ & $98^{*} 92$ \\
\hline 6 & ${ }^{90} \mathrm{Zr}$ & 163 & $8^{*} 5$ & $0 * 70$ & $0^{*} 20$ & $0 * 07$ & 603 & $0^{*} 52$ & $146^{*} 52$ \\
\hline 7 & ${ }^{90} \mathrm{Zr}$ & 400 & $14 * 4$ & $1^{*} 20$ & $0^{*} 70$ & $0=15$ & $5 \times 38$ & $0^{*} 46$ & $112^{*} 11$ \\
\hline 8 & ${ }^{118} \mathrm{Sn}$ & 163 & $9=4$ & $1 * 90$ & $0^{*} 15$ & $0^{*} 02$ & 6.63 & $1 * 40$ & $232^{*} 01$ \\
\hline
\end{tabular}

The value of real nuclear phase-shift parameter $\mu / 4 \Delta$ lies in the domain $0^{*} 02 \leq \mu / 4 \Delta \leq 0^{*} 3$ for $\pi^{+}$and in the domain $0^{*} 02 \leq \mu / 4 \Delta \leq 0^{*} 28$ for $\pi^{-}$. The value of the nuclear phase shift parameter $\mu / 4 \Delta$ has a tendency to decrease with an increase in mass number.

It is evident from our studies that the value of $\mathrm{R}$ increases as the target mass increases, for the same energy, as expected. Fig. 5 shows a relationship between $\mathrm{R}$ and $\mathrm{A}^{1 / 3}$. The least square method determines a linear relation of the from, 


$$
\mathrm{R}=1 * 119 \mathrm{~A}^{1 / 3}+0 * 689
$$

The surface diffuseness ' $d$ ' is calculated from SAM parameters and are shown in tables -1 and 2 . The surface diffuseness remains roughly constant in the study. Fig. 6 shows a linear relation between $\mathrm{d}$ and $\mathrm{A}^{1 / 3}$

$$
\mathrm{d}=-0^{*} 028 \mathrm{~A}^{1 / 3}+0^{*} 635
$$

The total reaction cross-section $\sigma_{R}$ has been calculated using the equation and the value of $\sigma_{R}$ are presented in tables -1 and 2 . The value of $\sigma_{R}$ increases as the target mass increases when the beam energy remains the same. It is well known that $\sigma_{R}$ depends also on the beam energy. Fig.7 shows a plot of $\sigma_{R}$ against $A^{1 / 3}$. The least squares method determines a linear relation of the form, $\sigma_{R}=31^{*} 720 \mathrm{~A}^{1 / 3}-25^{*} 243$.
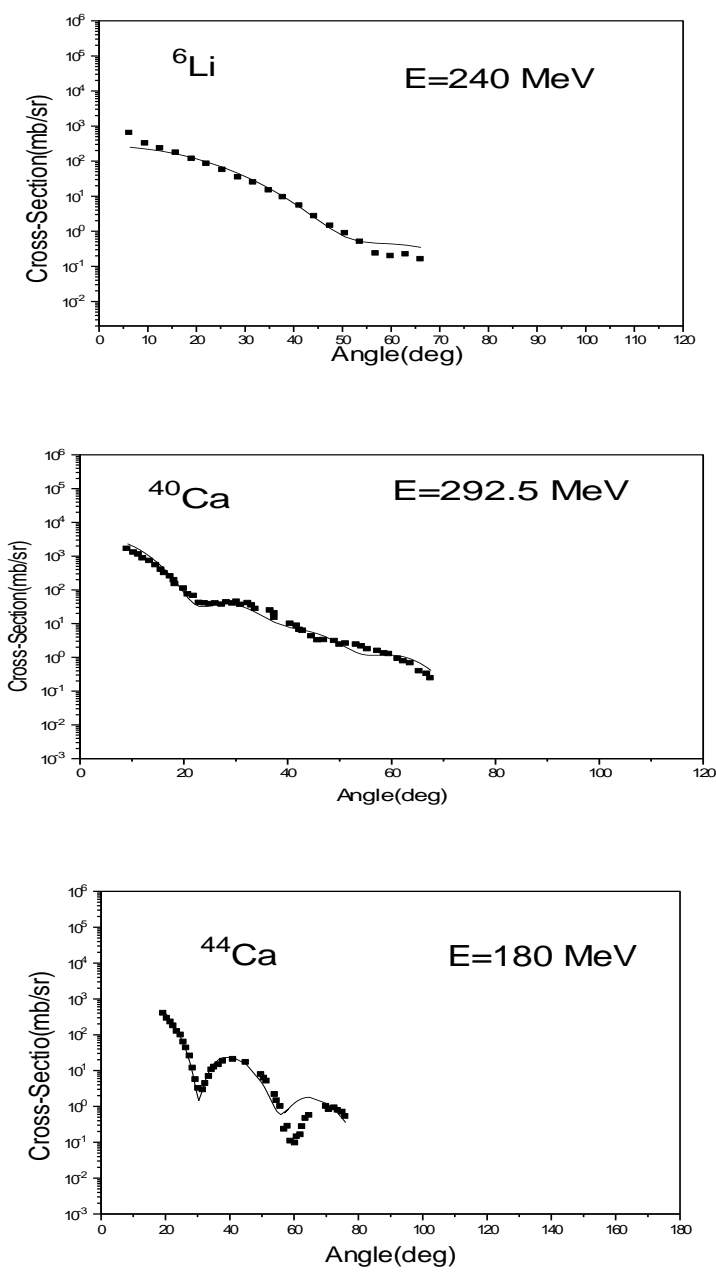

Fig.1: SAM analyses of $\pi^{+}$-particles from ${ }^{6} \mathrm{Li},{ }^{40,44} \mathrm{Ca}$.

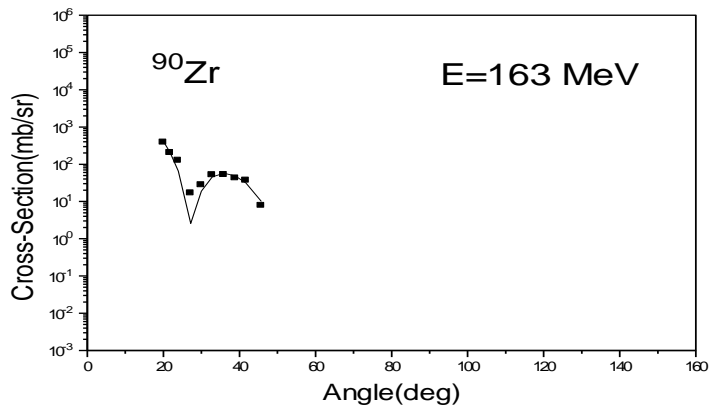



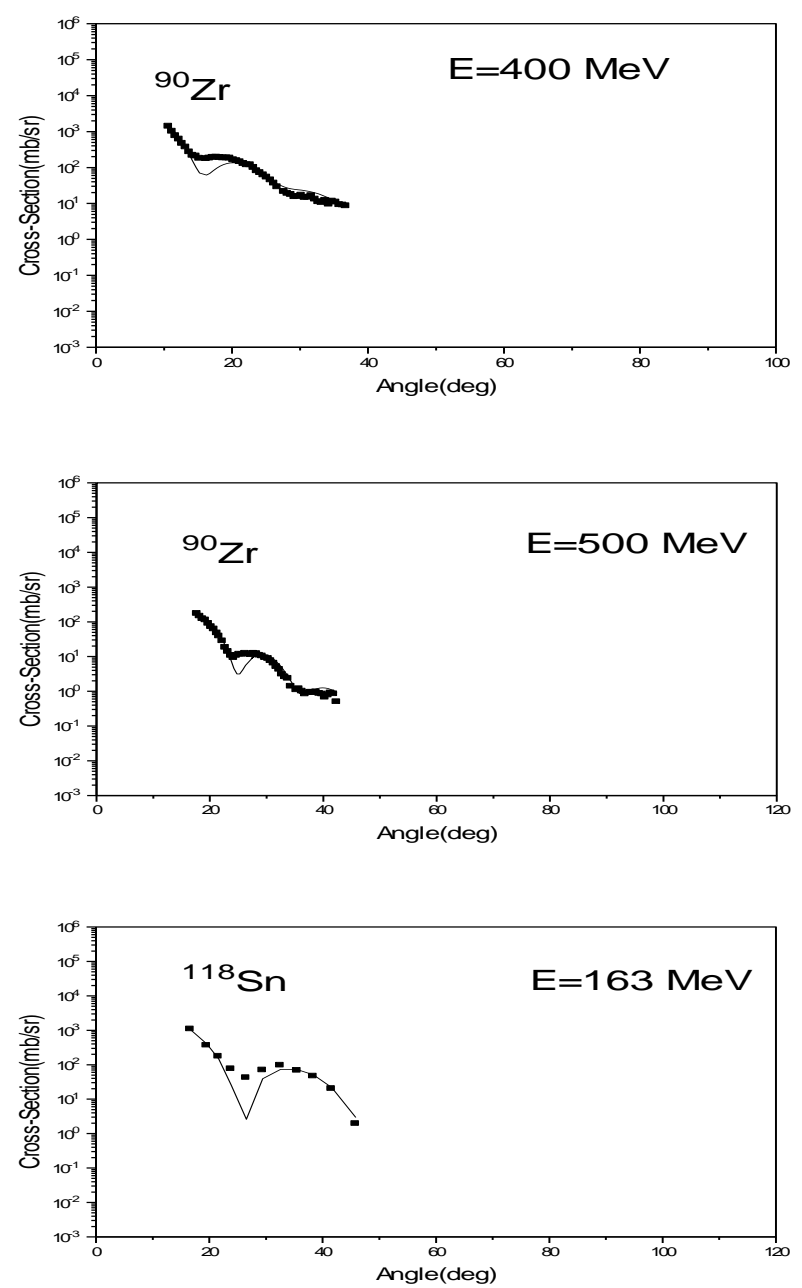

Fig.2: SAM analyses of $\pi^{+}$-particles from ${ }^{90} \mathrm{Zr},{ }^{118} \mathrm{Sn}$.
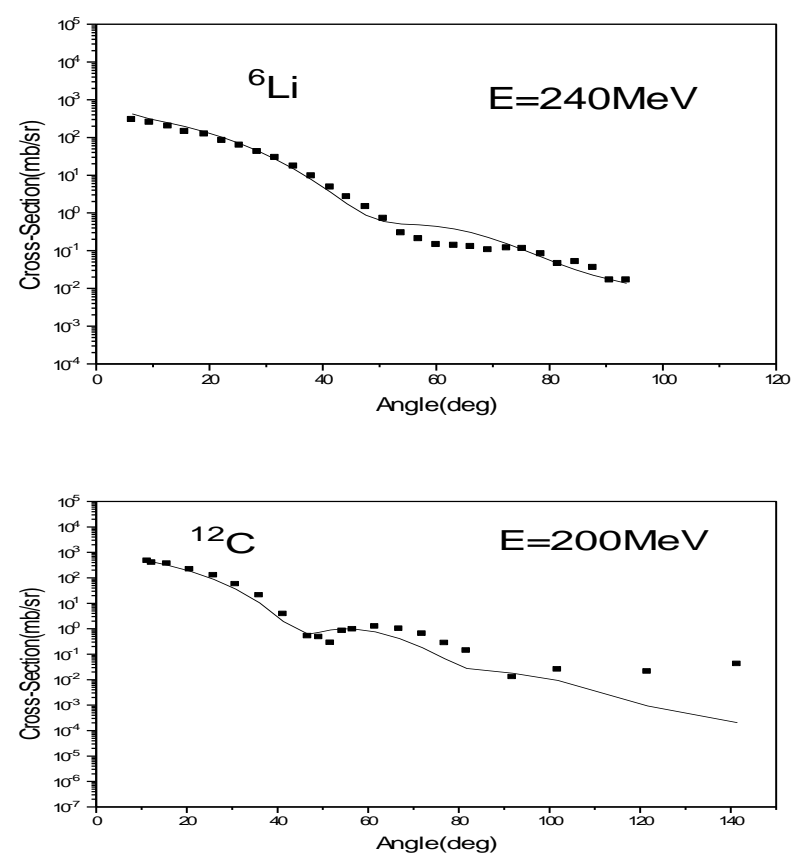

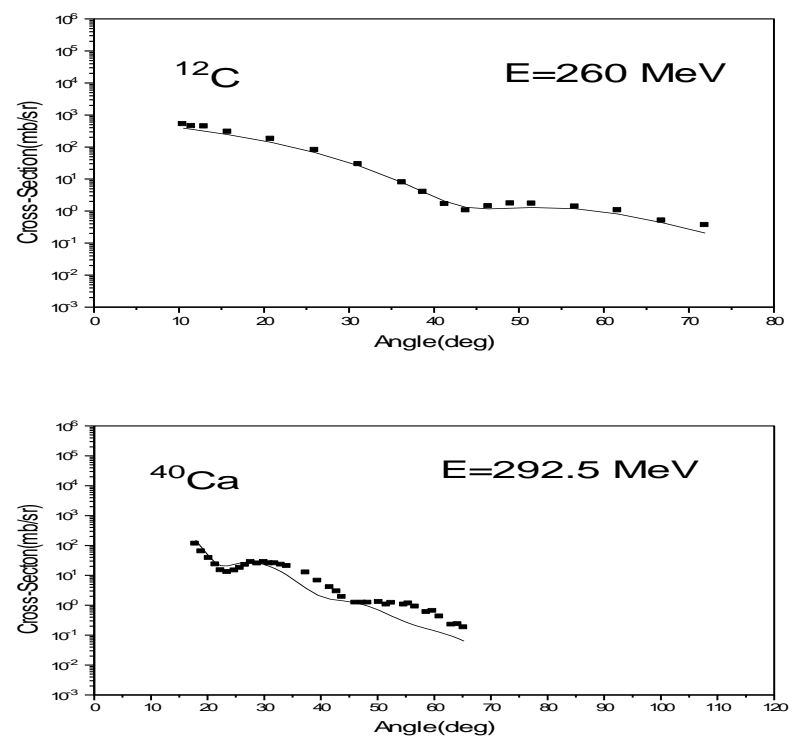

Fig.3: SAM analyses of $\pi^{-}$- particles from ${ }^{6} \mathrm{Li},{ }^{12} \mathrm{C},{ }^{40} \mathrm{Ca}$
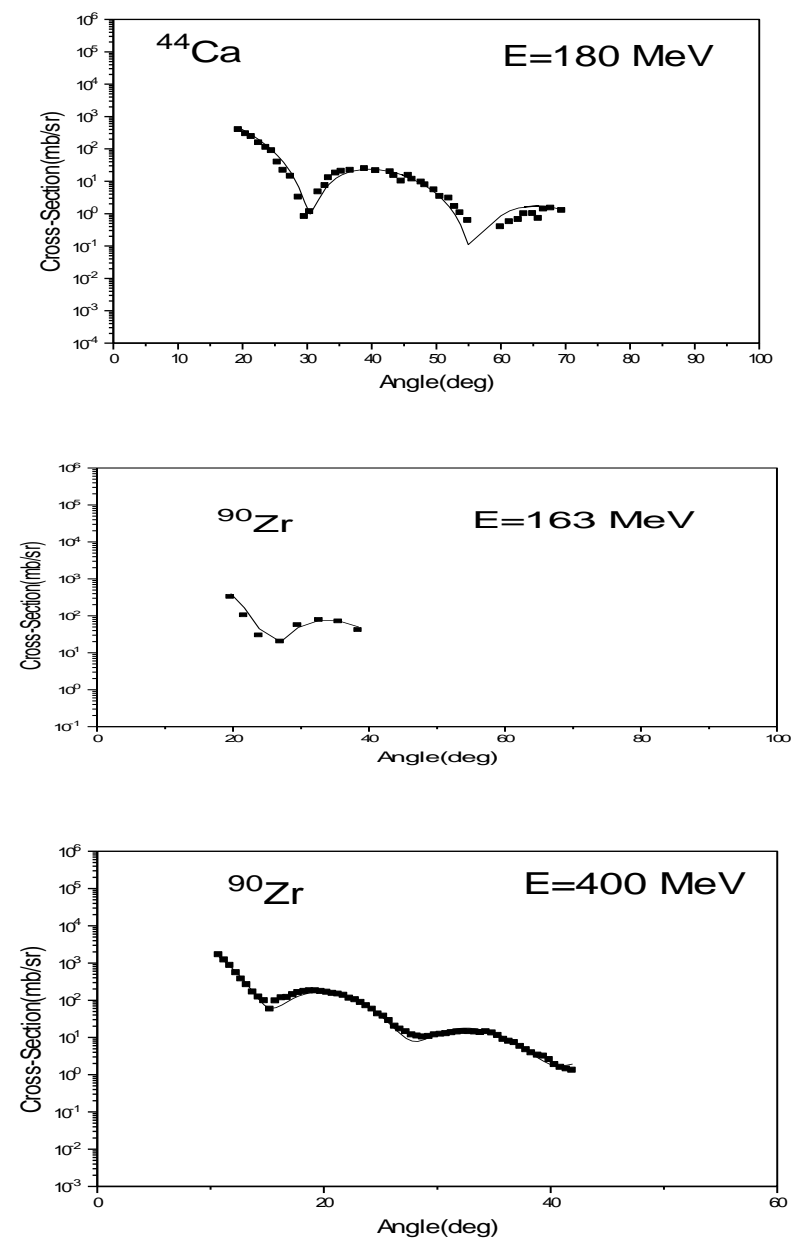


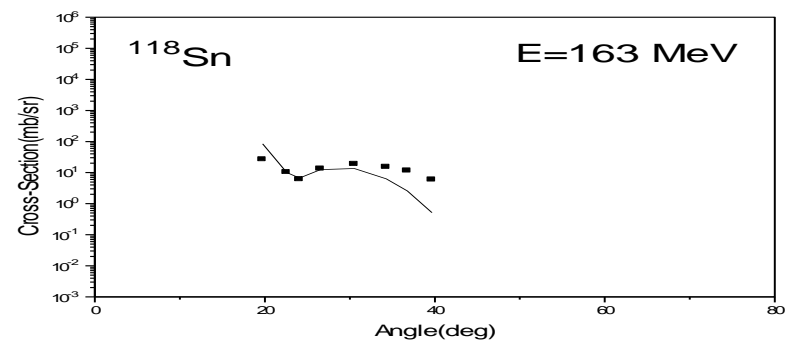

Fig.4: SAM analyses of $\pi$ particles from ${ }^{44} \mathrm{Ca},{ }^{90} \mathrm{Zr},{ }^{118} \mathrm{Sn}$.

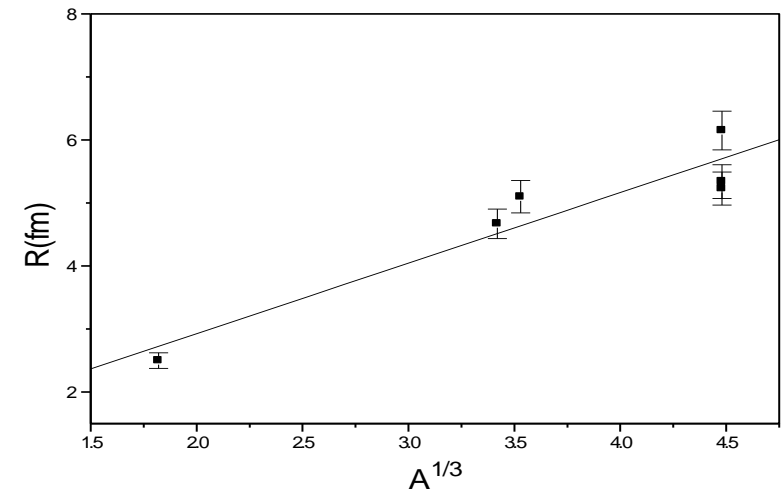

Fig 6: Plot of interaction radius $\mathrm{R}(\mathrm{fm})$ versus $\mathrm{A}^{1 / 3}$ for $\pi^{+}$.

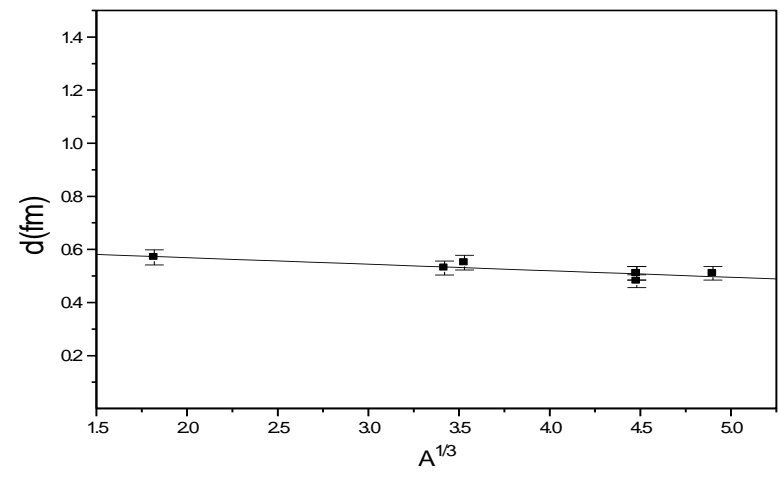

Fig 7: Plot of surface diffuseness versus $A^{1 / 3}$ for $\pi^{+}$

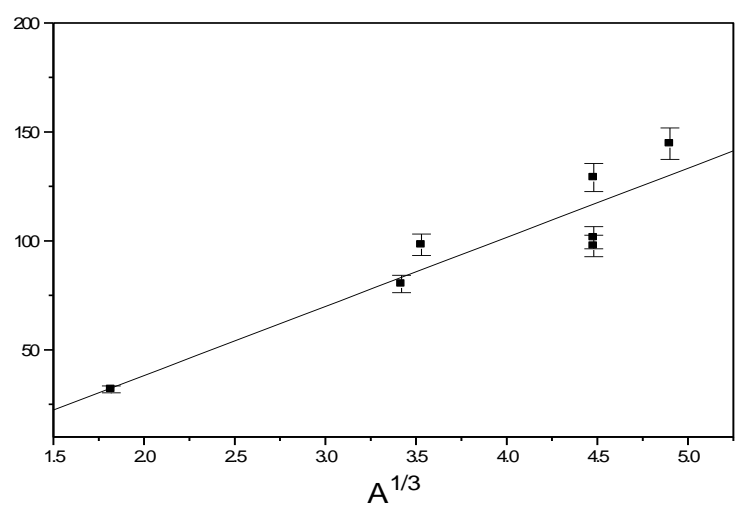

Fig.8: Plot of reaction cross-section $\sigma_{R}(\mathrm{mb})$ versus $\mathrm{A}^{1 / 3}$ for $\pi^{+}$. 


\section{IV. conclusion}

The three parameters of SAM formalism for Frahn and Venter can reasonably well account for the elastic scattering of pions, $\pi^{+}$and $\pi^{-}$. The angular distributions of elastic scattering of $\pi^{+}$and $\pi^{-}$at different energies 163 to $500 \mathrm{MeV}$ from several nuclei between ${ }^{6} \mathrm{Li}$ and ${ }^{118} \mathrm{Sn}$ have been analyzed with the SAM formalism and the best fit parameters $\Lambda, \Delta$ and $\mu$ have been obtained. The quality of SAM fit is poor at low beam energies and at larger angles, while in heavier nuclei a fairly good agreement is obtained. The agreement between the experiment and theory is reasonable and quite satisfactory. The SAM is thus a successful model, the parameters are well unique and physically meaningful and simplicity is its advantage.

\section{References}

[1]. W.E. Frahn and R.H. Venter Ann. Phys (N.Y.), 24, 243(1963).

[2]. W.E. Frahn and R.H. Venter Ann. Phys (N.Y.), 27, 385(1964).

[3]. K.R. Greider and Glassgold, Ann. Phys., 10, 100 (1960).

[4]. A.S. Shalay, M.Y.H. Hasan and M.M.H. Et.al. Phys Rev. A2, 37, (2007)

[5]. N. Austern and J.S. Blair, Ann. Phys. (N.Y), 33, 15(1965).

[6]. J.M. Potgieter and W.E. Frahn, Nucl. Phys. Rev. ,C 35, 931,(1987)

[7]. A. BUdzanowski, H. Dabrow, L. Freindl, K. Grotowski, S. Micek, R. Planeta and A. Strazalowski Phys. Rev., C17, 951, (1978).

[8]. P.Sing, A.Chaterjee, S.K. Gupta and S.S. Kerekatte. Phys. Rev., C43, 1867 (1991)

[9]. A. Akheizer and I. Pomerchuk, J. Phycis (USSR), 9, 741, (1945).

[10]. H.M. Sen Gupta, M. Rahman, H.Z. Rahman and S. Kari Nuovo cim. , 15A, 751 (1973).

[11]. Wallress and Ford, Phys.Rev. , 97, 726, (1955).

[12]. L.R.B. Elton, Introductory nuclear theory, p-287, (1959).

[13]. J.L. Ullman ET. al. Prc35, 1099, (1987).

[14]. D.R. Sarker et al., Int. J. Mod. Phys., E 13(2), 505, (2004). 